\title{
Accuracy of incoherent Speckle Tracking for circular Gaussian Signals
}

\author{
Francesco De Zan
}

\begin{abstract}
This paper provides a formula for the accuracy of incoherent speckle tracking (intensity cross-correlation) of homogeneous patches. The result is based on the determination of the curvature of the cross-correlation function and the noise which affects its first derivative.
\end{abstract}

Index Terms-Synthetic Aperture Radar, SAR interferometry, delay estimation, coherent cross-correlation, speckle tracking

\section{INTRODUCTION}

The availability of SAR data with high resolution justifies the interest in non-interferometric methods for estimating relative shifts between images. These methods are often based on the cross-correlation of images. Examples of application are found in the area of deformation monitoring, especially for motions in the along-track direction, to which interferometry is blind. Moreover, cross-correlation methods are also used as tools to fine co-register images for interferometry or to assist phase unwrapping.

In theory, the best estimator for the shifts is the maximization of the coherent cross-correlation, but in some cases there are limitations caused by the need to compensate the interferometric phases prior to cross-correlation. An alternative is to use methods based on spectral diversity [1], [2], which achieve very high efficiency [3], or to cross-correlate intensities, i.e. the squared magnitudes of the focused signals. In this last case the phases become completely irrelevant. It is usual to refer to this technique as "incoherent speckle tracking" or simply "speckle tracking". This paper derives the theoretical accuracy of speckle tracking, assuming homogeneous patches and an arbitrary coherence level.

The performance for coherent cross-correlation has been given in [4], [3], [5] and is reported in this paper for comparison. The coherent multi-image case has been treated in [6]: the equivalent for the incoherent case is still missing. This paper deals with the case of two images and incoherent crosscorrelation.

\section{MODEL AND PERFORMANCE}

\section{A. Circular Gaussian signals}

Homogeneous patches of fully-developed speckle are modeled as independent circular Gaussian samples. The master image samples will be indicated with $x_{n}$ and the slave samples with $y_{n}$. It is supposed that the original complex images are critically sampled. Since the intensities necessitate a doubled spectral support, the complex images have to be oversampled

The author is with the DLR (German Aerospace Center), Oberpfaffenhofen, D-82230 Wessling, Germany. e-mail:francesco.dezan@dlr.de with a factor two before computing the intensities. The interpolated samples will carry semi-integer indices: for example the new sample between $x_{0}$ and $x_{1}$ will be called $x_{1 / 2}$.

The function $c(\xi)$ will be the cross-correlation of master and slave image intensities, with the following definition

$$
\begin{aligned}
& c(\xi)=\sum_{2 n \in \mathcal{S}}\left|x_{n}\right|^{2}\left|y_{n}(\xi)\right|^{2} \\
& \text { with } \mathcal{S}=\mathbb{Z} \cap(-N, N] .
\end{aligned}
$$

The summation is performed over integer and semi-integer $n$ 's. The total number of independent samples (for the original complex images) is $N$, but the sum has $2 N$ terms. The $y_{n}(\xi)$ is a delayed version of the slave, i.e. a resampling:

$$
y_{n}(\xi)=\sum_{k \in \mathbb{Z}} y_{k} \operatorname{sinc}(n+\xi-k)
$$

this time summing only over integer $k$ 's. The delay $\xi$, a real variable, shifts the entire sequence $y_{n}$.

The sampling interval is normalized to 1 and all the results will have to be scaled to the system resolution. The signals are also normalized to have unitary power

$$
\mathrm{E}\left[\left|x_{n}\right|^{2}\right]=\mathrm{E}\left[\left|y_{n}\right|^{2}\right]=1,
$$

so that the expected value of $x_{n} \bar{y}_{n}$ is simply the coherence: $\mathrm{E}\left[x_{n} \bar{y}_{n}\right]=\gamma(\bar{z}$ indicates the complex-conjugate of $z)$. The coherence is considered to be real in the following, with no substantial consequences.

\section{B. The accuracy of correlation maximization}

The estimation of the delay is obtained by maximizing the correlation, i.e. finding the delay $\xi$ for which the correlation (1) attains its maximum:

$$
\hat{\xi}=\underset{\xi}{\arg \max }\{c(\xi)\},
$$

that is also a point where the first derivative $c^{\prime}(\xi)$ annihilates. Without loss of generality, in the rest of the paper the true value of the delay is assumed to be zero.

The accuracy of the determination of the delay depends on the curvature of the correlation peak, i.e. the average slope of $c^{\prime}(\xi)$ at zero, and on the variance of $c^{\prime}(\xi)$ at the same point [4]. The estimation uncertainty is given by

$$
\sigma_{I}^{2}=\operatorname{Var}[\hat{\xi}]=\frac{\operatorname{Var}\left[c^{\prime}(0)\right]}{\mathrm{E}\left[c^{\prime \prime}(0)\right]^{2}}
$$

which can be understood as the variance of the zero-crossing position of $c^{\prime}(\xi)$. 
With the definition in (1) the expected value of the correlation is found to be

$$
\begin{aligned}
\mathrm{E}[c(\xi)]= & 2 N \mathrm{E}\left[\left|x_{n}\right|^{2}\left|y_{n}(\xi)\right|^{2}\right] \\
= & 2 N\left(\mathrm{E}\left[x_{n} \bar{x}_{n}\right] \cdot \mathrm{E}\left[y_{n}(\xi) \bar{y}_{n}(\xi)\right]\right. \\
& \left.+\mathrm{E}\left[x_{n} \bar{y}_{n}(\xi)\right] \cdot \mathrm{E}\left[y_{n}(\xi) \bar{x}_{n}\right]\right) \\
= & 2 N\left(1+\gamma^{2} \operatorname{sinc}^{2}(\xi)\right) .
\end{aligned}
$$

This derivation is an application of Reed's theorem [7]. The first and second derivatives follow easily:

$$
\begin{aligned}
\mathrm{E}\left[c^{\prime}(\xi)\right] & =4 N \gamma^{2} \operatorname{sinc}(\xi) \operatorname{sinc}^{\prime}(\xi) \\
\mathrm{E}\left[c^{\prime \prime}(\xi)\right] & =4 N \gamma^{2}\left(\operatorname{sinc}(\xi) \operatorname{sinc}^{\prime \prime}(\xi)+\operatorname{sinc}^{2}(\xi)\right) .
\end{aligned}
$$

Thanks to linearity, expected values and derivatives can be interchanged, so that (8) represents also the average slope of the first derivative and the slope of the average first derivative. At the point of interest $\xi=0$ we have

$$
\begin{aligned}
\mathrm{E}\left[c^{\prime}(0)\right] & =0 \\
\mathrm{E}\left[c^{\prime \prime}(0)\right] & =-4 N \gamma^{2} \pi^{2} / 3 .
\end{aligned}
$$

Equation (10) provides the denominator of (5). Concerning the numerator, the derivation of the variance of the first derivative of $c(\xi)$ is more complicated and is left for the appendix. The result, valid for large $N$, is

$$
\operatorname{Var}\left[c^{\prime}(0)\right]=\mathrm{E}\left[\left|c^{\prime}(0)\right|^{2}\right]=N \frac{8 \pi^{2}}{15}\left(2+5 \gamma^{2}-7 \gamma^{4}\right)
$$

Finally, making use of (10) and (11) it is possible to write a closed-form expression for the performance of the crosscorrelation of speckle intensities:

$$
\begin{aligned}
\sigma_{I} & =\frac{\sqrt{\mathrm{E}\left[\left|c^{\prime}(0)\right|^{2}\right]}}{\left|\mathrm{E}\left[c^{\prime \prime}(0)\right]\right|} \\
& =\sqrt{\frac{3}{10 N}} \frac{\sqrt{2+5 \gamma^{2}-7 \gamma^{4}}}{\pi \gamma^{2}} .
\end{aligned}
$$

For comparison, this is the expression for the coherent crosscorrelation [3]:

$$
\sigma_{C}=\sqrt{\frac{3}{2 N}} \frac{\sqrt{1-\gamma^{2}}}{\pi \gamma}
$$

Both $\sigma_{I}$ and $\sigma_{C}$ are normalized to the resolution element; the number of independent samples $N$ refers to the original critically-sampled complex signals.

\section{Simulations, COMPARisOns, AND Limitations}

Numerical simulations with a large number of samples confirm the overall validity of expression (13). Figure 1 shows the normalized accuracy of the shift estimation: the standard deviation of the estimates is multiplied by the square root of the number of samples. Formally this is equivalent to setting $N=1$ in (13).

The solid line shows the prediction according to (13) and the stars represent the corresponding simulations. The dashed line represents the coherent cross-correlation (14), the triangles the simulations.
The same figure reports also the performance for the crosscorrelation of amplitude signals (only simulations). The performance is worse than the intensity correlation for low coherences, but becomes almost indistinguishable for coherences larger than 0.6. It is possible that the amplitude signal requires even more oversampling, because of the non-linearity of the square root.

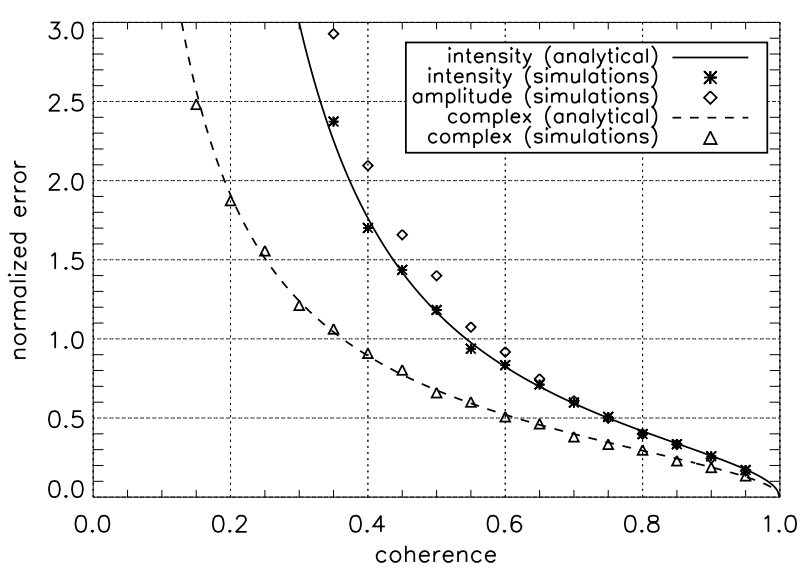

Fig. 1. The normalized accuracy $(\sigma \sqrt{N})$ of cross-correlation as a function of coherence for complex signals, amplitudes and intensities.

\section{A. Limitations}

The result presented in this letter is valid in the case of a large number of samples, similarly to a Cramér-Rao bound. For practical purposes, it is interesting to analyze the behavior for a finite number of samples.

The validity limitations come mostly from a typical threshold effect which appears at low signal-to-noise ratio and small number of samples. Analogous situations happen, e.g., in frequency estimation [8] or in phase unwrapping and they cannot be characterized studying only the local properties of the maximum as it is done in this paper.

In the case of the cross-correlation, simulations indicate that to have a performance within $1 \mathrm{~dB}$ from (13), one needs roughly 1000 samples if the coherence is $0.3,200$ samples if the coherence is 0.5 , and 50 if it is 0.7 . This can be understood in terms of the probability of confusing the main lobe of the cross-correlation function with a secondary lobe. Looking at the denominator of (13), the condition for being within the main peak with high probability is $\gamma^{2} \sqrt{N} \gg 1$. For coherent cross-correlation less samples are required, since the same condition leads to $\gamma \sqrt{N} \gg 1$ (see also [4]). The total number of independent samples counts for the threshold effect, even if the estimation patch is extended in both azimuth and range directions. It must be said that sometimes, in order to select the correct peak, a-priori information can be used.

Another limitation affects high coherences and it is a border effect. For $\gamma=1$ the formula (13) yields $\sigma_{I}=0$, but for finite $N$ the error is never zero. The problem can be almost entirely avoided by normalizing the energy of the signals in the correlation window for each delay (the estimator is then 
called normalized cross-correlation [9]). However, this effect is not likely to show up in typical situations, in which incoherent cross-correlation is used for medium to low coherences.

It is worth noting that the homogeneity hypothesis may lose validity when the cross-correlation window grows too much. It is difficult to provide figures for this additional limitation, which will depend both on the intrinsic homogeneity of the scene and the system resolution.

\section{B. Comparison with coherent cross-correlation}

In [4] it was observed, thanks to simulations, that the performance of incoherent speckle tracking is about 2 times worse $(3 \mathrm{~dB})$ than the coherent cross-correlation for high coherences. It is now possible to state more precisely that the performance degradation $\sigma_{I}^{2} / \sigma_{C}^{2}$ tends to $9 / 5=1.8$ in the limit $\gamma \rightarrow 1$. As suggested by one reviewer, the slight difference could be due to the increased robustness of the intensity signal to the noise, compared to the real and imaginary parts alone. At high coherences the noise is relevant only when the speckle is small, but for the intensity signal to be small it is necessary that both real and imaginary parts are small.

For lower coherences the loss w.r.t the coherent case is larger than $3 \mathrm{~dB}$ : e.g. for $\gamma=0.5$ we obtain $\sigma_{I}^{2}=3 \sigma_{C}^{2}$. In this case the same mechanism mentioned above would work against the signal: since the noise is comparable or larger than the signal, the intensity ends up privileging noise over signal. This discussion should only be taken as an intuitive explanation for what has been observed.

\section{CONCLUSION}

This letter has investigated the performance of shift estimation based on intensity cross-correlation, in the case of homogeneous speckle patches. Simulations confirm the analytic derivation and some practical validity limitations have been discussed. Different from coherent cross-correlation, incoherent cross-correlation is probably not the maximum-likelihood estimator and the performance obtained in this paper is not a Cramér-Rao bound.

\section{APPENDIX}

To compute the noise on the derivative one needs to evaluate $\mathrm{E}\left[\left|c^{\prime}(\xi)\right|^{2}\right]$ at $\xi=0$. One starts by writing explicitly the first derivative (over-bars stand for complex-conjugate):

$$
\begin{aligned}
c^{\prime}(0) & =\left.\frac{d}{d \xi} c(\xi)\right|_{\xi=0}=\left.\sum_{2 n \in \mathcal{S}}\left|x_{n}\right|^{2} \frac{d}{d \xi}\left|y_{n}(\xi)\right|^{2}\right|_{\xi=0} \\
& =\sum_{2 n \in \mathcal{S}}\left|x_{n}\right|^{2}\left(y_{n} \partial \bar{y}_{n}+\bar{y}_{n} \partial y_{n}\right)
\end{aligned}
$$

with the shorthand notation

$$
\begin{aligned}
\partial y_{n} & =\left.\frac{d}{d \xi} y_{n}(\xi)\right|_{\xi=0}=\left.\sum_{k \in \mathbb{Z}} y_{k} \frac{d}{d \xi} \operatorname{sinc}(n+\xi-k)\right|_{\xi=0} \\
& =\sum_{k \in \mathbb{Z}} y_{k} \operatorname{sinc}^{\prime}(n-k) .
\end{aligned}
$$

The sum is over integer $k$ 's but $n$ can be semi-integer. The squared derivative is then the double sum

$$
\begin{aligned}
& \left|c^{\prime}(0)\right|^{2}= \\
& \quad \sum_{2 n \in \mathcal{S}}\left|x_{n}\right|^{2}\left(y_{n} \partial \bar{y}_{n}+\bar{y}_{n} \partial y_{n}\right) \cdot \sum_{2 k \in \mathcal{S}}\left|x_{k}\right|^{2}\left(y_{k} \partial \bar{y}_{k}+\bar{y}_{k} \partial y_{k}\right)
\end{aligned}
$$

which originates four terms:

$$
\begin{aligned}
& T_{1}(n, k)=\left|x_{n}\right|^{2}\left|x_{k}\right|^{2} y_{n} y_{k} \partial \bar{y}_{n} \partial \bar{y}_{k} \\
& T_{2}(n, k)=\left|x_{n}\right|^{2}\left|x_{k}\right|^{2} y_{n} \bar{y}_{k} \partial \bar{y}_{n} \partial y_{k} \\
& T_{3}(n, k)=\left|x_{n}\right|^{2}\left|x_{k}\right|^{2} \bar{y}_{n} y_{k} \partial y_{n} \partial \bar{y}_{k} \\
& T_{4}(n, k)=\left|x_{n}\right|^{2}\left|x_{k}\right|^{2} \bar{y}_{n} \bar{y}_{k} \partial y_{n} \partial y_{k} .
\end{aligned}
$$

One can see immediately that $T_{1}(n, k)=\bar{T}_{4}(n, k)$ and $T_{2}(n, k)=\bar{T}_{3}(n, k)$, but since they will be found to be real, the equalities hold without the need to complex-conjugate.

The expected values are derived by applying Reed's theorem [7] on $\mathrm{E}\left[T_{1}(n, k)\right]$ and $\mathrm{E}\left[T_{2}(n, k)\right]$. Each expected value generates a sum of $4 !=24$ terms, which correspond to the possible permutations of the tuples $\left(x_{n}, x_{k}, y_{n}, y_{k}\right)$ and $\left(x_{n}, x_{k}, y_{n}, \partial y_{k}\right)$. Each term is then the product of four correlations. The permutations and the resulting terms are listed in Tables I and II. The symbols

$$
\begin{aligned}
A_{n k} & =\mathrm{E}\left[y_{n} \bar{y}_{k}\right]=\mathrm{E}\left[x_{n} \bar{x}_{k}\right]=\operatorname{sinc}(n-k) \\
B_{n k} & =-\mathrm{E}\left[y_{n} \partial \bar{y}_{k}\right]=\mathrm{E}\left[y_{k} \partial \bar{y}_{n}\right]=\operatorname{sinc}^{\prime}(n-k) \\
C_{n k} & =\mathrm{E}\left[\partial y_{n} \partial \bar{y}_{k}\right]=\sum_{m \in \mathbb{Z}} \operatorname{sinc}^{\prime}(m) \operatorname{sinc}^{\prime}(m-(n-k)) \\
& =-\operatorname{sinc}^{\prime \prime}(n-k)
\end{aligned}
$$

are introduced for convenience. Asterisks replace irrelevant factors: for instance, the first row of Table I represents the computation of the term

$$
\mathrm{E}\left[x_{n} \bar{x}_{n}\right] \mathrm{E}\left[x_{k} \bar{x}_{k}\right] \mathrm{E}\left[y_{n} \partial \bar{y}_{n}\right] \mathrm{E}\left[y_{k} \partial \bar{y}_{k}\right]=1 \cdot 1 \cdot 0 \cdot 0
$$

but the first two factors can be ignored since they will be anyway multiplied by zero. Many of the terms are indeed zero and the final results of the sum are:

$$
\begin{aligned}
\mathrm{E}\left[T_{1}(n, k)\right]= & -B_{n k}^{2}\left(1+A_{n k}^{2}\right)\left(1+2 \gamma^{2}+\gamma^{4}\right) \\
\mathrm{E}\left[T_{2}(n, k)\right]= & \left(1+3 \gamma^{2}\right) A_{n k} C_{n k}+\left(1+\gamma^{2}\right) A_{n k}^{3} C_{n k} \\
& -\left(\gamma^{2}+\gamma^{4}\right) A_{n k}^{2} B_{n k}^{2}
\end{aligned}
$$

At this point it is possible to sum over $n$ and $k$ to obtain the expected value of (19). The sums are for integer and semiinteger values in the interval $-N / 2, N / 2$, however one of the two sums can be extended between plus and minus infinity. In this way we avoid border effects and the formulas obtained are valid for large $N$ (sums over finite $N$ do not yield nice formulas, but allow us to study numerically the impact of $N$ on border effects). We compute preliminarily the following series, summing over integer and semi-integer $k$ :

$$
\begin{aligned}
& \sum_{2 k \in \mathbb{Z}} A_{n k}^{2} B_{n k}^{2}=2 \pi^{2} / 15 \\
& \sum_{2 k \in \mathbb{Z}} B_{n k}^{2}=\sum_{2 k \in \mathbb{Z}} A_{n k} C_{n k}=2 \pi^{2} / 3 \\
& \sum_{2 k \in \mathbb{Z}} A_{n k}^{3} C_{n k}=2 \pi^{2} / 5 .
\end{aligned}
$$




\begin{tabular}{cccc|c}
$\bar{x}_{n}$ & $\bar{x}_{k}$ & $\partial \bar{y}_{n}$ & $\partial \bar{y}_{k}$ & \\
\hline$x_{n}$ & $x_{k}$ & $y_{n}$ & $y_{k}$ & $* \cdot * \cdot 0 \cdot 0$ \\
$x_{n}$ & $x_{k}$ & $y_{k}$ & $y_{n}$ & $1 \cdot 1 \cdot B_{n k} \cdot\left(-B_{n k}\right)$ \\
$x_{n}$ & $y_{n}$ & $x_{k}$ & $y_{k}$ & $* \cdot * \cdot * \cdot 0$ \\
$x_{n}$ & $y_{n}$ & $y_{k}$ & $x_{k}$ & $* \cdot * \cdot * \cdot 0$ \\
$x_{n}$ & $y_{k}$ & $x_{k}$ & $y_{n}$ & $1 \cdot \gamma \cdot \gamma B_{n k} \cdot\left(-B_{n k}\right)$ \\
$x_{n}$ & $y_{k}$ & $y_{n}$ & $x_{k}$ & $* \cdot * \cdot 0 \cdot 0$ \\
$x_{k}$ & $x_{n}$ & $y_{n}$ & $y_{k}$ & $* \cdot * \cdot 0 \cdot 0$ \\
$x_{k}$ & $x_{n}$ & $y_{k}$ & $y_{n}$ & $A_{n k} \cdot A_{n k} \cdot B_{n k} \cdot\left(-B_{n k}\right)$ \\
$x_{k}$ & $y_{n}$ & $x_{n}$ & $y_{k}$ & $* \cdot * \cdot 0 \cdot 0$ \\
$x_{k}$ & $y_{n}$ & $y_{k}$ & $x_{n}$ & $A_{n k} \cdot \gamma A_{n k} \cdot B_{n k} \cdot\left(-\gamma B_{n k}\right)$ \\
$x_{k}$ & $y_{k}$ & $x_{n}$ & $y_{n}$ & $* \cdot * \cdot 0 \cdot *$ \\
$x_{k}$ & $y_{k}$ & $y_{n}$ & $x_{n}$ & $* \cdot * \cdot 0 \cdot *$ \\
$y_{n}$ & $x_{n}$ & $x_{k}$ & $y_{k}$ & $* \cdot * \cdot *$ \\
$y_{n}$ & $x_{n}$ & $y_{k}$ & $x_{k}$ & $* \cdot * \cdot 0 \cdot 0$ \\
$y_{n}$ & $x_{k}$ & $x_{n}$ & $y_{k}$ & $* \cdot 0$ \\
$y_{n}$ & $x_{k}$ & $y_{k}$ & $x_{n}$ & $\gamma \cdot 1 \cdot B_{n k} \cdot\left(-\gamma B_{n k}\right)$ \\
$y_{n}$ & $y_{k}$ & $x_{n}$ & $x_{k}$ & $* \cdot 0 \cdot 0$ \\
$y_{n}$ & $y_{k}$ & $x_{k}$ & $x_{n}$ & $\gamma \cdot \gamma A_{n k} \cdot \gamma B_{n k} \cdot\left(-\gamma B_{n k}\right)$ \\
$y_{k}$ & $x_{n}$ & $x_{k}$ & $y_{n}$ & $\gamma A_{n k} \cdot A_{n k} \cdot \gamma B_{n k} \cdot\left(-B_{n k}\right)$ \\
$y_{k}$ & $x_{n}$ & $y_{n}$ & $x_{k}$ & $* \cdot * \cdot 0 \cdot 0$ \\
$y_{k}$ & $x_{k}$ & $x_{n}$ & $y_{n}$ & $* \cdot * \cdot 0 \cdot *$ \\
$y_{k}$ & $x_{k}$ & $y_{n}$ & $x_{n}$ & $* \cdot * \cdot 0 \cdot *$ \\
$y_{k}$ & $y_{n}$ & $x_{n}$ & $x_{k}$ & $* \cdot * \cdot 0 \cdot 0$ \\
$y_{k}$ & $y_{n}$ & $x_{k}$ & $x_{n}$ & $\gamma A_{n k} \cdot \gamma A_{n k} \cdot \gamma B_{n k} \cdot\left(-\gamma B_{n k}\right)$
\end{tabular}

TABLE I

COMPUTATION OF E[ $\left.T_{1}(n, k)\right]$. THE RESUlT IS THE SUM OF THE TERMS IN THE RIGHT COLUMN. ON THE LEFT, THE CORRESPONDING PERMUTATIONS OF THE TUPLE $\left(x_{n}, x_{k}, y_{n}, y_{k}\right)$.
Incidentally, thanks to the infinite sums, the dependence on $n$ vanishes too.

With the help of (27), (28) and (29)-(31) we finally obtain:

$$
\begin{aligned}
& \mathrm{E}\left[\left|c^{\prime}(0)\right|^{2}\right] \\
& =\sum_{2 n \in \mathcal{S}}\left\{\sum_{2 k \in \mathbb{Z}} 2 \mathrm{E}\left[T_{1}(n, k)\right]+2 \mathrm{E}\left[T_{2}(n, k)\right]\right\} \\
& =2 \sum_{2 n \in \mathcal{S}}\left\{-\left(1+2 \gamma^{2}+\gamma^{4}\right)\left(2 \pi^{2} / 3+2 \pi^{2} / 15\right)\right. \\
& \quad+\left(1+3 \gamma^{2}\right) 2 \pi^{2} / 3+\left(1+\gamma^{2}\right) 2 \pi^{2} / 5 \\
& \left.\quad-\left(\gamma^{2}+\gamma^{4}\right) 2 \pi^{2} / 15\right\} \\
& =2 N \cdot 2 \frac{2 \pi^{2}}{15}\left(2+5 \gamma^{2}-7 \gamma^{4}\right),
\end{aligned}
$$

which is Eq. (11).

\section{AKNOWLEDGEMENT}

The author would like to thank the two anonymous reviewers for their work, especially for inquiring about the validity of the result when the number of samples is finite.

\section{REFERENCES}

[1] S.N. Madsen and H.A. Zebker, "Automated absolute phase retrieval in across-track interferometry," in International Geoscience and Remote Sensing Symposium (IGARSS), May 1992, vol. 2, pp. 1582 -1584.

[2] R. Scheiber and A. Moreira, "Coregistration of interferometric SAR images using spectral diversity," Geoscience and Remote Sensing, IEEE Transactions on, vol. 38, no. 5, pp. 2179 -2191, Sep 2000.

[3] R. Bamler and M. Eineder, "Accuracy of differential shift estimation by correlation and split-bandwidth interferometry for wideband and delta-k SAR systems," IEEE Geosci. Remote Sensing Letters, vol. 2, pp. 151155, Apr 2005.

[4] R. Bamler, "Interferometric stereo radargrammetry: Absolute height determination from ERS-ENVISAT interferograms," Proc. IGARSS 2000, vol. 2, pp. 742-745, Jul 2000.

[5] W. Walker and G. Trahey, "A fundamental limit on delay estimation using partially correlated speckle signals," Ultrasonics, Ferroelectris and Frequency Control, IEEE Transactions on, vol. 42, pp. 301-308, Mar 1995.

[6] F. De Zan, "Coherent shift estimation for stacks of SAR images," Geoscience and Remote Sensing Letters, IEEE, vol. 8, no. 6, pp. 1095 -1099 , Nov 2011.

[7] I. Reed, "On a moment theorem for complex Gaussian processes," Information Theory, IRE Transactions on, vol. 8, no. 3, pp. $194-195$, Apr 1962.

[8] D. Rife and R. Boorstyn, "Single tone parameter estimation from discretetime observations," Information Theory, IEEE Transactions on, vol. 20, no. 5, pp. 591 - 598, Sep 1974.

[9] A. Monti Guarnieri and C. Prati, "SAR interferometry: a "quick and dirty" coherence estimator for data browsing," Geoscience and Remote Sensing, IEEE Transactions on, vol. 35, no. 3, pp. 660 - 669, May 1997.

TABLE II

COMPUTATION OF $\mathrm{E}\left[T_{2}(n, k)\right]$. THE RESUlt IS THE SUM OF THE TERMS IN THE RIGHT COLUMN. ON THE LEFT, THE CORRESPONDING PERMUTATIONS OF THE TUPLE $\left(x_{n}, x_{k}, y_{n}, \partial y_{k}\right)$ 